\title{
ILCEA
}

Revue de l'Institut des langues et cultures

d'Europe, Amérique, Afrique, Asie et Australie

$26 \mid 2016$

Mémoire, vérité et justice en Uruguay

\section{Las investigaciones históricas de la Universidad de la República}

University of Republic's Historical Investigations on the Dictatorship and State

Terrorism in Uruguay

Les investigations historiques de l'Université de la République sur le terrorisme

d'État en Uruguay

\section{Carla Larrobla y Fabiana Larrobla}

\section{OpenEdition}

\section{Journals}

Edición electrónica

URL: http://journals.openedition.org/ilcea/3950

DOI: 10.4000/ilcea.3950

ISSN: 2101-0609

Editor

UGA Éditions/Université Grenoble Alpes

Edición impresa

ISBN: 978-2-84310-334-6

ISSN: 1639-6073

Referencia electrónica

Carla Larrobla y Fabiana Larrobla, «Las investigaciones históricas de la Universidad de la República », ILCEA [En línea], 26 | 2016, Publicado el 07 julio 2016, consultado el 01 mayo 2019. URL : http:// journals.openedition.org/ilcea/3950; DOI : 10.4000/ilcea.3950

Este documento fue generado automáticamente el 1 mayo 2019.

(C) ILCEA 


\title{
Las investigaciones históricas de la Universidad de la República
}

\author{
University of Republic's Historical Investigations on the Dictatorship and State \\ Terrorism in Uruguay \\ Les investigations historiques de l'Université de la République sur le terrorisme \\ d'État en Uruguay
}

Carla Larrobla y Fabiana Larrobla

\section{Las investigaciones históricas}

1 En setiembre de 2005, se conformó un equipo de 17 investigadores, Álvaro Rico convocó al equipo que se encontraba trabajando de forma honoraria como grupo auxiliar ${ }^{1}$ al trabajo que se encontraban realizando los arqueólogos en la búsqueda de restos de detenidos desaparecidos en los predios del Batallón n. ${ }^{\circ} 13$.

2 Los primeros pasos del equipo consistieron en la revisación de los documentos que se encontraban en la entonces Secretaría de seguimiento de la Comisión para la Paz, sobre todo de los Legajos personales de personas desaparecidas que constituyeron la base para la elaboración de las Fichas Personales de cada una de ellas. Además del acceso a la recopilación de testimonios que poseía la Secretaría y la organización Madres y Familiares de detenidos desparecidos.

Al mismo tiempo se gestionó el ingreso del equipo a algunos archivos estatales que se encontraban (y encuentran) restringidos al uso público. Algunos de esos archivos consultados fueron: Dirección Nacional de Información e Inteligencia, Dirección Nacional de Migración (Ministerio del Interior), archivos del Ministerio de Relaciones Exteriores, archivo de historias clínicas de Sanidad Militar (Ministerio de Defensa Nacional), archivo de expedientes de la Justicia Militar en custodia de la Suprema Corte de Justicia (Oficina de archivos judiciales), archivo de la Comisión para la Paz, Archivo General de la Nación (Sección Judicial), Poder Legislativo ${ }^{2}$. Por otro lado, accedimos a archivos de organizaciones sociales y educativas como el Archivo del Centro de Estudios 
Interdisciplinarios Uruguayos ${ }^{3}$, el Archivo de la Desaparición Forzada de Personas de Madres y Familiares de Uruguayos Detenidos Desaparecidos, y el Archivo Sara Méndez - Raúl Olivera.

El Equipo de historiadores publicó ${ }^{4}$ en el año 2007 la primera investigación académica en cuatro tomos (un quinto tomo contenía el Informe Final del equipo de arqueólogos-GIAF), que sistematizó la dimensión de la desaparición forzada y delitos conexos en el Uruguay, y que completó, a través de la elaboración de las Fichas Personales de las víctimas, los datos correspondientes al total de los $\mathbf{1 7 0}$ detenidos desaparecidos ${ }^{5}$ documentados hasta ese momento en el Informe Final de la Comisión para la Paz (abril 2003). Las fichas incorporan varias dimensiones, una breve información personal, un apartado sobre la detención y desaparición de la persona y en algunos casos se enmarca la detención en la oleada y operativo represivo que corresponda. Se incorporó toda la documentación encontrada que refiriera a la persona y se reconstruyó el periplo de gestiones y denuncias realizadas por la familia o terceros en aras de encontrarla. Estas fichas buscaron recuperar la trayectoria biográfica de la víctima, desde su militancia hasta su desaparición, pero también se intentó dar cuenta de los esfuerzos realizados para encontrar su paradero primero y para buscar justicia después.

5 También se reconstruyeron los contextos represivos y operativos desplegados por el Estado uruguayo, en algunos casos, en coordinación con fuerzas represivas de Argentina, entre 1971 (antes del golpe de Estado) y 1982 (año en que se constata la última desaparición forzada en el Uruguay). Los operativos represivos dan cuenta de las distintas dinámicas represivas desplegadas desde el Estado uruguayo y cómo se articularon coordinaciones con los gobiernos dictatoriales del Cono Sur. En este último caso, también se presenta un informe sobre la gestación y el desarrollo del Plan Cóndor, en tanto la mayoría de las desapariciones se enmarcan en operaciones de coordinación regional.

Esta primera etapa de trabajo se cierra con la mencionada publicación de los 5 tomos, luego la investigación sobre Detenidos Desaparecidos será retomada en el año 2009.

\section{Las investigaciones históricas sobre la dictadura y el terrorismo de Estado en Uruguay}

7 Durante la primera etapa, la investigación histórica del equipo de historiadores no sólo se concentró en el fenómeno de la desaparición forzada sino que recopiló documentación sobre distintas dimensiones del terrorismo de Estado: el asesinato político, la prisión política masiva y prolongada (metodología represiva que caracterizó al régimen autoritario en Uruguay), las políticas de vigilancia y persecutorias desplegadas en contra del movimiento sindical y estudiantil y los partidos políticos, así como también se obtuvo documentación ilustrativa del control y represión ejercidas sobre la sociedad civil y sobre los uruguayos que se encontraban exilados en distintos países.

Dicha acumulación permitió proseguir con la investigación y preparar un nuevo trabajo que permitiera presentar una visión global de los efectos que el terrorismo de Estado generó en la sociedad uruguaya. La Investigación histórica sobre la Dictadura y el Terrorismo de Estado en Uruguay (2009) editada en 3 tomos por la Universidad de la República y presentada a comienzos del año 2010, contiene, por primera vez en Uruguay, una recopilación documental sobre esas distintas dimensiones del terrorismo de Estado y sus 
víctimas que proporcionan una visión de conjunto del terrorismo de Estado, abriendo la posibilidad de nuevas investigaciones en distintos terrenos del pasado reciente.

Uno de los resultados de este primer trabajo resultó la comprobación del universo de víctimas durante la dictadura por la causal del asesinato político o muerte por responsabilidad del Estado y sus agentes especializados, bajo la modalidad de torturas, enfermedades no tratadas, suicidios en situación carcelaria, castigos de distinto tipo, así como el universo de presos y presas políticas en los distintos establecimientos carcelarios y cuarteles del país en el período dictatorial. En el primer caso, se trata de 116 asesinados políticos entre 1973-1984. La investigación sobre asesinados políticos por responsabilidad del Estado, será profundizada por el equipo en otra etapa del trabajo realizado bajo la órbita de la UdelaR y Presidencia de la República entre los años 2011 y 2014 que abordaremos más adelante.

10 En lo que se refiere a la prisión masiva y prolongada se confeccionó una lista inicial y abierta que da cuenta de la existencia de $\mathbf{5 9 2 5}$ presos políticos varones recluidos en el Establecimiento Militar de Reclusión n. ${ }^{\circ} 1$-Penal de Libertad-y de 739 mujeres (registro hasta el año 1979) en el Establecimiento Militar de Reclusión n. ${ }^{\circ} 2$-Punta de Rieles-, así como 186 presas políticas recluidas en el interior del país -cárcel de Paso de los Toros. Estas listas fueron confeccionadas en base a la información oficial encontrada en los archivos estatales por lo que representa un listado incompleto pero da cuenta del fenómeno en rasgos generales. Allí figuran quiénes fueron procesados por la Justicia Militar, existieron muchos uruguayos más que fueron detenidos en distintas dependencias policiales y militares pero liberados antes de ser sometidos a ésta.

11 En otro apartado se da cuenta de la Represión contra los menores de edad y las diversas situaciones por las que atravesaron las víctimas: detención y desaparición, nacimiento en cautiverio, cambio de identidad y apropiación ilegítima, menores presos con sus progenitores (67 casos documentados). De estos casos documentados, tres menores de edad se encuentran detenidos desaparecidos hasta el presente (Beatriz y Washington Hernández Hobbas de 17 y 15 años respectivamente y Carlos Severo de 16 años, los tres desaparecidos en la Argentina). Cuatro casos tratan de bebés nacidos en cautiverio, posteriormente desaparecidos y que luego fueron recuperados. Doce casos refieren a niños y adolescentes, cuyos padres se encuentran desaparecidos y que fueron secuestrados temporariamente y posteriormente recuperaron su identidad. Un caso pertenece a un bebé nacido en prisión, de madre detenida y padre desaparecido, entregado a su familia biológica. Cuatro son los casos cuyas madres fueron detenidasdesaparecidas embarazadas y cuyos nacimientos no han podido ser confirmados. Se registran dos casos de detenidas desaparecidas probablemente embarazadas al momento de su detención. Ocho casos hacen referencia a niños que fueron abandonados forzadamente en el momento del secuestro de sus padres y que fueron recuperados por su familia. Cinco niños fueron trasladados en forma ilegal junto con sus madres desde la Argentina y liberados en Uruguay y, finalmente, el último caso da cuenta de un niño secuestrado, cuyo padre es detenido y posteriormente es localizado por su familia.

En el Tomo II se aborda el tema de las violaciones a los derechos civiles ilustrando un aspecto poco documentado aún en las investigaciones sobre el período dictatorial: La vigilancia a la sociedad civil (el «insilio»). Al respecto, se aporta documentación oficial sobre el intento de «control total» de la población por la dictadura: la represión a la cultura (censura de libros, cine, teatro, carnaval, música, conferencias, artistas e intelectuales), clausura de medios de comunicación, control de la educación, seguimiento 
a las actividades religiosas, vigilancia a diversas organizaciones de la sociedad civil. La vigilancia a todos los espacios de nucleamiento social que aparecen representados en esta sección, ilustrados a través de ejemplos que van desde el control a una reunión de Damas de Tupperware hasta un campamento de los Boy Scout.

Por su parte y en lo que refiere a las actividades culturales varios documentos dan cuenta de cómo operaban los servicios de Inteligencia, controlando que los dictámenes de la Comisión de censura fueran respetados. De esta manera encontramos seguimientos a agrupaciones carnavalescas, vigilancia en tablados, censura de películas y asistencia del personal de la DNII a cines y teatros tanto para controlar los espectáculos como las reacciones del público presente.

mente se incorpora un apartado sobre distintas formas de colaboración de sectores de la población con el régimen dictatorial, para lo cual fueron seleccionadas distintas modalidades de colaboración que van desde la denuncia anónima hasta aquellas que se realizaban de forma personal en las oficinas de Inteligencia. Cabe destacar que todas las denuncias activaban el inicio de operativos que, por más pequeños que resultasen, involucraban a toda una comunidad de individuos. Se comienza por la solicitud de antecedentes del denunciado, por el allanamiento de su domicilio, el interrogatorio a sus vecinos, su posterior detención, etc. Estos mecanismos que se inician luego de una denuncia van fortaleciendo la cultura del miedo y de la delación. De esta manera la dictadura ataca los lazos sociales, intentando romper las relaciones sociales basadas en la solidaridad y la confianza.

En la última sección del presente libro se desarrolla el tema del Exilio, en particular, la vigilancia en el exterior del país a las actividades de solidaridad, las organizaciones y los ciudadanos. Esta vigilancia se extendía fuera de fronteras, una vez que los ciudadanos partían del país la vigilancia continuaba, principalmente a través del Ministerio de Relaciones Exteriores y el Departamento II (Exterior) del Servicio de Información de Defensa. Asimismo se aporta documentación sobre el control, detenciones y expulsiones de Extranjeros residentes en el país.

También se aborda el tema de las dimensiones y etapas de la Represión al movimiento sindical y la CNT, reconstruyéndose un listado general de 891 sindicalistas presos durante la dictadura En otro apartado se estudia la Represión a la enseñanza y los estudiantes, en particular, contra la Universidad de la República y la Federación de Estudiantes Universitarios del Uruguay, la Enseñanza Secundaria y la Universidad del Trabajo. Subdividimos esta sección, en la represión a la universidad y los universitarios, por un lado, y la represión en la enseñanza secundaria y la UTU por otro.

Merece particular atención los resultados de la investigación que se transcriben en el apartado sobre los cambios en la institucionalidad estatal bajo la dictadura, la configuración de un Estado «clandestino» a través del accionar de los servicios de inteligencia y la coordinación represiva regional y el papel de la justicia militar en el juzgamiento y condena a civiles.

18 Finalmente, el Tomo III resume y actualiza los datos referidos a los avances procesados por la sociedad uruguaya desde la recuperación de la democracia en el país (1985) en materia de verdad, justicia, memoria y reparación a las víctimas del terrorismo de Estado. En ese sentido, se repasa la formación de Comisiones investigadoras y los informes oficiales, las leyes aprobadas por el Parlamento y las convenciones y protocolos internacionales incorporados a la legislación nacional, decretos y resoluciones del Poder 
Ejecutivo, antecedentes, sentencias y autos de procesamiento dictados por la Justicia Penal contra responsables de la violación de los derechos humanos y crímenes de lesa humanidad cometidos bajo la dictadura.

\section{Nueva etapa de trabajo: desde el 2011 a la actualidad}

Desde el 2009 y hasta 2011 el equipo trabajó en una nueva actualización de la investigación que culminó con la publicación de la misma el 1 de noviembre de 2011. Esta nueva actualización, focalizada en los casos de los detenidos desaparecidos supuso la incorporación de nueva documentación a las fichas personales ya que se contó con nuevos archivos.

Por otra parte se elaboraron dos tipos de cronologías para reforzar documentalmente los operativos represivos contra las organizaciones políticas ya mencionadas. La cronología de hechos (que intenta dar cuenta de la continuidad de la represión y de su simultaneidad en diversos países) y la cronología de documentos que supone la difusión de una gran cantidad de documentación elaborada por los servicios de inteligencia sobre la organizaciones.

21 Entre la nueva documentación incorporada o encontrada, vale destacar, por un lado, las Fichas Patrónimicas de OCOA (Organismo Coordinador de Operaciòn Antisubversivas) y del SID (Servicio de Información de Defensa), y por otro, nuevos documentos encontrados de gran relevancia, como por ejemplo: incautación de materiales en domicilios de detenidos desaparecidos en las fechas próximas a su detención en Argentina, demostrando que las fuerzas uruguayas actuaban en el país vecino con total libertad.

Durante la presidencia de José Mujica, la secretaría pasó a denominarse «Secretaría para los Derechos Humanos del Pasado Reciente» y se produjo una ampliación de sus tareas, lo que supuso que el equipo de historiadores dirigiera su trabajo hacia distintos frentes, que pasamos a detallar:

- Revisión de archivos: se continúa con la revisión de la documentación al tiempo que un equipo de archivólogos trabaja en su ordenación y sistematización.

La gran dimensión de algunos archivos, como el de la DNII o el proveniente del MDN, implican que aún hoy se continúe encontrando material que permite profundizar el conocimiento sobre las dinámicas represivas y seguir acumulando información sobre las víctimas. El archivo de Sanidad Militar, permitió encontrar necropsias y registros de ciudadanos muertos en prisión o en enfrentamientos de los cuales no se tenían datos oficiales al respecto de su defunción. También los expedientes de la Justicia Militar aportaron valiosa información sobre los operativos represivos donde murieron o fueron asesinadas algunas de las víctimas.

- Se responden oficios y solicitudes de la justicia y de organismos estatales. Eso implica la realización del contexto en que la víctima murió o desapareció así como también se realiza una breve explicación de los documentos que se solicitan.

- Se continuó con el trabajo de investigación histórica que no solo se avoca a la actualización de los casos y contextos de desaparición sino que al incorporar la dimensión de asesinados políticos, se abre un nuevo universo de trabajo. En este sentido es importante señalar que por resolución de la Presidencia de la República, el período de tiempo sobre el que se investiga se amplió hasta 1968, dando cuenta también de cómo la represión y el terrorismo de Estado se profundizaron con anterioridad al Golpe de Estado. 


\section{Última actualización: 28 de febrero de 2015} investigación sobre el asesinato político se estructura en torno a dos ejes considerados inseparables: por un lado el contexto histórico en el que se producen dichos crímenes (contemplado en la primera sección anteriormente descripta) y por otro lado el eje referido a la dimensión individual de la víctima, concretándose en la elaboración de las fichas personales, las que contienen información referida a los datos biográficos, de militancia y entornos represivos de cada caso particular.

Fueron confeccionadas 124 fichas personales de ciudadanos asesinados y/o fallecidos por responsabilidad del Estado uruguayo y constituye una investigación en curso, en la medida en que las cifras no pueden ser consideradas definitivas y considerando que en la actualidad se continúa investigando sobre casos sobre los que aún no se conoce con certeza la circunstancia de muerte. Sobre el período 1968-1973 sólo pudo construirse un listado de ciudadanos fallecidos, siendo ésta una investigación en curso.

La tercera sección refiere a la actualización de las fichas personales sobre Detenidos desaparecidos. En este caso hubo modificaciones y avances importantes con respecto a la investigación publicada en el año 2007. En primer lugar se modificó el original ordenamiento por orden alfabético de las fichas personales y se adoptó un criterio cronológico por fecha de detención y/o secuestro (al igual que en las fichas personales de asesinados políticos). La fundamentación de este cambio reside en que el ordenamiento 
anterior, que además discriminaba por países según el momento y lugar de detención, no permitía visualizar con claridad ni las oleadas represivas ni las estrategias utilizadas por las agencias en sus intentos por desarticular las organizaciones políticas. Por otra parte la división por países tampoco permitía dar cuenta del despliegue de la coordinación represiva en la región en el marco, la mayoría de las veces, del Plan Cóndor. Es así que una persona podía ser detenida en un país, trasladada a otro y desaparecida finalmente en otro país del Cono Sur.

El agrupamiento de las detenciones por fechas hace visible, entonces, los momentos de mayor concentración de la represión y permite abordar el fenómeno de la desaparición forzada a través del tiempo, pudiendo relacionarla con otras estrategias represivas desplegadas en la región, basadas en el asesinato y/o la detención y posterior encarcelamiento de los ciudadanos.

Por otra parte, las fichas personales incorporaron no sólo nueva información documental, sino que modificaron su estructura, a la vez que fueron incorporados nuevos casos denunciados en Uruguay y otros países de la región.

La cuarta sección presenta los casos de 13 niños apropiados y recuperados y 4 casos de adolescentes no recuperados, 3 de los cuales continúan desaparecidos, en tanto que el restante fue recuperado su cuerpo seis años más tarde de su secuestro.

Esta sección también presenta información sobre casos de mujeres detenidas estando embarazadas sin que aún se haya confirmado el nacimiento y destino de sus hijos.

La quinta sección está referida a los casos de N.N. hallados en las costas uruguayas entre los años 1975 y 1979. En ésta se presentan las fichas de diez restos identificados, así como las fichas de 20 restos que aún no han podido identificarse.

En suma, los aportes más importantes de esta nueva actualización pueden sintetizarse de la siguiente manera:

1. Modificaciones en el criterio de ordenación de las fichas personales (Detenidos desaparecidos y Asesinados políticos) lo que nos permite visualizar el despliegue represivo en la región así como las oleadas represivas contra las organizaciones políticas;

2. Ampliación de los operativos represivos incorporando la dimensión del asesinato político y de aquellos procedimientos policiales y militares que tuvieron como resultado la detención y posterior encarcelamiento de militantes;

3. Sobre los casos de Detenidos desaparecidos. Hasta el momento fue posible constatar la desaparición de 192 ciudadanos, incorporándose seis nuevos casos que no habían sido denunciados en Uruguay hasta el momento:

FILIPAZZI, Rafaela Juliana (Legajo CONADEP, reconocida como víctima por la Comisión de la Verdad en Paraguay);

POTENZA, José (Legajo CONADEP, reconocido como víctima por la Comisión de la Verdad en Paraguay);

LEMA AGUIAR, Miguel Ángel (reconocido en la Rpca. Argentina);

GATTI, Adriana (asesinada en Argentina en 1977, con cuerpo desaparecido y enterrado como NN, reconocido en 1983);

RANDO, Francisco (Legajo CONADEP y Secretaría de Derechos Humanos, Rpca. Argentina);

ALTAMIRANO ALZA, Ricardo (cuerpo desaparecido en la Argentina en 1976 y enterrado como NN, recuperado por el EAAF en 2010). 
4. Sobre el asesinato político. La investigación realizada hasta febrero de 2015 abordó en primera instancia el período comprendido entre 1973 y 1985, determinando hasta el momento 85 casos de ciudadanos fallecidos por responsabilidad del Estado.

Sobre el período 1968-1973, la investigación confeccionó una lista de ciudadanos fallecidos en el marco de la represión estatal, que asciende a los 124 casos.

Los resultados de esta investigación permiten advertir que el universo de víctimas, tanto de desaparición forzada como de asesinato político, continúa siendo parcial y provisorio, ya que existen nuevas denuncias en curso así como casos en los que aún no se cuenta con información suficiente como para poder dilucidar las circunstancias de muerte.

9 A lo largo de 10 años de trabajo, entre el 2005 y el 2015, el equipo de historiadores procuró realizar una investigación rigurosa y seria, que permitiera visualizar el mundo de las víctimas del terrorismo de Estado pero también las distintas dimensiones que éste desplegó sobre la sociedad uruguaya. La investigación ha permitido poner en manos de la sociedad un acervo documental que se encuentra accesible en la web y ha intentado sistematizar y organizar la información de forma clara y contundente. A lo largo de los años, se ha asistido a la justicia y a las causas reparatorias y se continúa trabajando en aras de alcanzar verdad y justicia.

Si bien el formato de la investigación puede resultar denso para un lector común, el objetivo de la misma ha sido poner a disposición del público, sea cual fuera su intención de consulta, una voluminosa información que permita acceder a los periplos recorridos por las víctimas del terrorismo de Estado en tanto individuos y seres políticos.

41 Por otra parte, la investigación siempre ha permanecido abierta, tanto para ser actualizada con nueva información y documentación, como para las investigaciones que puedan realizarse a partir de ella.

Porque la memoria y la historia están en permanente construcción. Y porque de todos depende que se continúe trabajando por verdad, justicia y Nunca Más.

\section{BIBLIOGRAFÍA}

RIco Álvaro (coord.) (2007), Investigación histórico sobre detenidos desaparecidos, 5 tomos, Montevideo: Presidencia de la República, IMPO.

RICo Álvaro (coord.) (2009), Investigación histórica sobre la dictadura y el terrorismo de Estado en Uruguay (1973-1985), Montevideo: Universidad de la República, Ediciones Cruz del Sur.

\section{NOTAS}

1. Jimena Alonso, Magdalena Figueredo, Mariana Iglesias, Carla Larrobla, Fabiana Larrobla.

2. Allí se utilizaron fundamentalmente los repartidos de la Comisión de Respeto a los Derechos Individuales, del Interior, de Defensa Nacional, de Asuntos Administrativos, entre otras, creadas por el Consejo de Estado. Allí se ubicaron fundamentalmente solicitudes de familiares, o incluso, 
en algunos casos de organizaciones sindicales que aun se mantenían en la legalidad, solicitando la liberación de algún detenido, por ejemplo.

3. Dependiente de la Facultad de Humanidades y Ciencias de la Educación, tiene entre otras la custodia de la Colección Marta Ponce de León-Ricardo Vilaró, Hugo Cores, Héctor Rodríguez y Daniel Baldassari. Si bien la mayor parte de sus documentos, son producto de organizaciones sociales o políticas, no brindaron un importante listado de militantes sindicales detenidos, por cuya libertad, se realizaron acciones en el exterior, fundamentalmente en Europa.

4. Ver <http://archivo.presidencia.gub.uy/_web/noticias/2007/06/2007060509.htm>.

5. Es importante aclarar que esta cifra fue aumentando a lo largo de los 10 años de trabajo del equipo. Como veremos en la última etapa, en número de detenidos desaparecidos asciende a 192.

\section{RESÚMENES}

En el siguiente texto se presenta el trabajo del equipo de Historiadores de la Universidad de la República en el marco del convenio con la Presidencia de la República para la realización de una investigación histórica sobre los crímenes cometidos bajo el Terrorismo de Estado durante la dictadura uruguaya entre 1973-1985. Se describe el recorrido de la investigación desde sus inicios en el año 2005 hasta la actualidad, dando cuenta de los principales resultados alcanzados.

In this paper, we present the findings of the group of historians working at the University of the Republic (Uruguay) as part of an agreement on historical research with the Presidency of the Republic, regarding the study of the crimes committed during State terrorism in the Uruguayan dictatorship (1973-1985). The research will be described since its inception in 2005 until today, highlighting the main results of the study.

Dans le texte suivant sont présentés les travaux du groupe d'historiens de l'Université de la République réalisés dans le cadre des accords avec la présidence de la République. Ces derniers concernent la réalisation d'une investigation historique sur les crimes commis dans le cadre du terrorisme d'État pendant la dictature uruguayenne entre 1973 et 1985 . Le processus d'investigation est décrit depuis ses débuts en 2005 jusqu'à l'actualité, en rendant compte de ses principaux résultats.

\section{ÍNDICE}

Keywords: Uruguay, dictatorship, State terrorism, history, memory

Palabras claves: Uruguay, dictadura, terrorismo de Estado, historia, memoria

Mots-clés: Uruguay, dictature, terrorisme d'État, histoire, mémoire

\section{AUTORES}

\section{CARLA LARROBLA}

Centro de Estudios Interdisciplinarios Uruguayos, Universidad de la República 


\section{FABIANA LARROBLA}

Centro de Estudios Interdisciplinarios Uruguayos, Universidad de la República 